\title{
Parental and job burnout in a Chinese sample
}

\author{
Wei Wang ${ }^{1} \cdot$ Shengnan Wang ${ }^{1} \cdot \mathrm{Xia} \mathrm{Liu}^{2} \cdot$ Yongxin $\mathrm{Li}^{1}$ (D)
}

Accepted: 11 February 2021 / Published online: 5 March 2021

(C) The Author(s) 2021

\begin{abstract}
Recently, parental burnout has received considerable attention. However, to what extent can parental burnout be distinguished from job burnout in general? Furthermore, what is the direction of the relationship between parental and job burnout? These questions remain to be explored. The present study sought to determine the relationship between parental and job burnout, examining the effects on fathers and the mothers sample separately. Study 1 aimed to provide further evidence of the distinction between parental and job burnout. In total, 597 students, 458 fathers, and 531 mothers took part in our survey. Furthermore, both parental and job burnout could positively predict their own depression $\left(\beta_{\text {father }}=0.27, p<0.01 ; \beta_{\text {mother }}=0.35, p<0.01\right)$. Parental burnout could significantly predict parenting-related variables (e.g., children's anxiety) $\left(\beta_{\text {father }}=0.23, p<0.01 ; \beta_{\text {mother }}=0.13\right.$, $p<0.01$ ), whereas job burnout could significantly predict job-related variables (e.g., job satisfaction) $\left(\beta_{\text {father }}=-0.27, p<0.01\right.$; $\left.\beta_{\text {mother }}=-0.28, p<0.01\right)$. These results support the distinction between parental and job burnout. In Study 2, a three-wave longitudinal design and a cross-lag model was established to infer the direction of the relationship. In total, 109 fathers and 144 mothers completed all questionnaires. The results showed that the previous parental burnout had a significant predictive effect on later job burnout consistently, whereas the previous job burnout did not show significant predictive effect on later parental burnout. The implications and necessary future research are also discussed.
\end{abstract}

Keywords Parental burnout $\cdot$ Job burnout $\cdot$ Distinction $\cdot$ Cross-lag model

\section{Introduction}

Children are an important part of marriage and family life, and daily experience with children can be a source of joy or pleasure for parents (Nelson, Kushlev, \& Lyubomirsky, 2014). However, parenting is also stressful work, and parental responsibilities and activities can be heavy burdens (Crnic \& Low, 2002). When parents do not have enough resources to

\section{Yongxin $\mathrm{Li}$}

liyongxin@henu.edu.cn

Wei Wang

sea_wangwei@163.com

Shengnan Wang

nicolesnwang@163.com

Xia Liu

liuxia@bnu.edu.cn

1 Institute of Psychology and Behavior, Henan University, Jinming Avenue, Kaifeng, Henan Province, China

2 Institute of Developmental Psychology, Beijing Normal University, No. 19 Xinkouwai Street, Beijing, China cope with daily parental stress, they may experience burnout (Roskam, Raes, \& Mikolajczaket, 2017), which includes a series of specific syndromes, such as feeling drained and emotionally distant from their children, having a reduced sense of accomplishment in parenting, and losing the pleasure of being with their children (Roskam, Brianda, \& Mikolajczaket, 2018).

Parental burnout may have negative effects on both parents themselves and their children. Burned out parents may feel that the parental work requires too much involvement and fell emotional drained by the parental role, they may do not have enough resource to coping the parental stress and try to escape from the situation. Mikolajczak, Brianda, Avalosse, and Roskam (2018) and Mikolajczak, Gross, and Roskam (2019a) indicated that parental burnout is positively related to suicidal and escape ideation, addiction, and sleep problems. Furthermore, exhausted parents may feel emotional distance from their children and become less involved in parenting work. Parental burnout may increase neglectful and violent behavior toward children (Mikolajczak et al., 2018; Mikolajczak, Gross, Stinglhamber, Norberg, \& Roskam, 2019b). Therefore, research on parental burnout is important.

The conceptualization and measurement of parental burnout have been deduced from those of job burnout. According 
to Maslach, Schaufeli, and Leiter (2001), job burnout is a psychological syndrome in response to the chronic interpersonal stressors of a job. In the early stages of burnout, many researchers have indicated that burnout is a work-related phenomenon (e.g., Freudenberger, 1974). When the Maslach Burnout Inventory (MBI) was developed (Maslach \& Jackson, 1981), the study of burnout as an academic concept spread all over the world (Igawa, 2018). At the same time, the concept of burnout expanded; for instance, marriage burnout (Pines, 1987) and parental burnout (Pelsma, 1989) emerged.

Parental burnout emerged in the 1980s; however, the concept has only recently become a subject of scientific interest. In the review of Sánchez-Rodríguez et al. (2019), they noted that there had been 39 articles regarding parental burnout since the first empirical study on the theme was published (i.e., Pelsma, 1989), most of which were published after 2010, especially after the pioneer work of Roskam et al. (2018), which developed the measurement of parental burnout. Their studies accelerated the research into parental burnout, and to date, parental burnout has received considerable attention from over 100 researchers across 40 countries (Mikolajczak et al., 2019b). However, with the rapid development of parental burnout, there are still some issues that remain to be explored-for instance, the relationship between parental burnout and related concepts, especially job burnout.

The conceptualization and measurement of job and parental burnout have some similarities. Historically, the definition of parental burnout was partly derived from the concept of job burnout: "Like job burnout, which results from exposure to chronic job stress, parental burnout is a specific syndrome resulting from enduring exposure to chronic parental stress" (Mikolajcak et al., 2018). Early studies on parental burnout also used existent job burnout scales, such as the MBI (Maslach \& Jackson, 1981), to assess parental burnout, simply changing the words "service subject" (e.g., Pelsma, 1989). Furthermore, in the development of measures of parental burnout, job burnout was usually selected as the criterion for a correlation analysis (e.g., Roskam et al., 2017, Kawamoto et al., 2018).

Though these two kinds of burnout are typically present simultaneously in the relevant research, each has its unique aspects, both in terms of conception and measurement (though job burnout is work-related and parental burnout is parentingrelated). Indeed, some scholars have noticed differences between these two concepts; for instance, as noted in their work on developing a measure of parental burnout, Roskam et al. (2018) argued that parents cannot "dehumanize" their children, so the depersonalization of job burnout may not be suitable in the parenting context. Furthermore, Mikolajczakl et al. (2019b) systematically examined the distinction between parental and job burnout in two separate studies. In the work of Mikolajczakl et al. (2019b), results of Study 1 supported the factorial distinctiveness of parental and job burnout.
Specifically, a two-order factor structure-6 first order factors (exhaustion, detachment, and inefficacy from MBI and PBI) loading on $2 \mathrm{~s}$ order factor (parental burnout and job burnout)fit the data better than other alterative models (including a two-order factor structure which 6 first order factors loading on a second order factor named burnout; and a two-order factor structure which 3 first order factor, exhaustion, detachment, and inefficacy loaded on $1 \mathrm{~s}$ order factor named burnout). Study 2 replicated the results of Study 1 and found that parental burnout could have more pronounced parentingrelated outcome variables (e.g., parental satisfaction), whereas job burnout had more pronounced job-related outcome variables (e.g., job satisfaction); both parental and job burnout could, however, predict general outcome variables (e.g., escape and suicidal ideation).

The above-mentioned study improved our understanding of parental burnout and its distinction from job burnout. However, there are still two questions that need to be further discussed. First, studies of their samples are all from Western countries, which limits the universality of the conclusions. The construct and the perception of Chinese family are quite different from Western. For instance, the "big family", which means three generations to live together is common in China, whereas this phenomenon is relative rare in the Western countries. Chinese emphases harmony in family and parents may regard parenting as their own duties and work. Therefore, the extent and effects of parenting burnout of Chinese parents may have some differences from that of the Western countries. It is necessary to discuss parental burnout in different cultures. Therefore, whether samples from a different culture could produce the same results should be examined. In addition, the direction of the relationship between parental and job burnout has not been examined. In other words, are burnedout parents more vulnerable to job burnout, or do parents with job burnout not have sufficient resources to take care of their children and so easily develop parental burnout?

As such, the purpose of the present study was to explore the relationship between parental and job burnout in two studies. Study 1, based on the pioneer work of Mikolajczak et al. (2019b), provided supplementary information about Chinese samples to distinguish between the concepts of parental and job burnout. Specifically, we examined whether the two types of burnout belong to different factor structures. Furthermore, we examined whether the two types of burnout can predict different outcome variables. In Study 2, we attempted to explore the direction of the relationship between parental and job burnout in the context of Chinese culture. Specifically, through a three-wave longitudinal design, a cross-lag model was established.

Moreover, the traditional Chinese perception of "men work outside; women perform housework inside" has lasted for thousands of years, which means that parenting is seen primarily as a mother's duty. In line with this opinion, parental 
burnout should only occur in the samples of mothers. However, in the past few decades, traditional values have been challenged by rapid social changes. An increasing number of women pay more attention to work-related duties (Zhang, 2010), while men are beginning to pay more attention to family responsibilities (Zou, Wu, \& Liu, 2016). In the context of rapid change, could mothers be at risk of experiencing both parental and job burnout? Could parental burnout also occur in the samples of father? Furthermore, is the relationship between parental and job burnout the same in the samples of mothers and fathers? Therefore, the present study divided our samples into fathers and mothers, and then examined the differences and the direction of the relationship between parental and job burnout.

\section{Study 1: Drawing a Distinction Between Parental and Job Burnout}

Study 1 was based on the work of Mikolajczak et al. (2019b); the factorial distinctiveness of parental and job burnout was examined in samples of fathers and mothers. If it is possible to distinguish parental burnout from job burnout, they would both have different loadings in a factor analysis. Furthermore, the predicted effects of parental and job burnout on job-related, general, and parenting-related outcome variables were examined. If it is possible to distinguish between parental and job burnout, then parental and job burnout would have different predicted consequence variables.

In the present study, three outcome variables were included, namely job satisfaction, children's anxiety, and depression. Job satisfaction was chosen because it is a job-related outcome and has previously been demonstrated to be a consequence of job burnout (e.g., Lizano \& Barak, 2015). Children's anxiety was chosen because it is a parentingrelated outcome; previous studies have indicated that parental burnout may increase parents' neglectful and violent behavior toward their children (Mikolajczaket al., 2018, 2019b), and that parental style is highly correlated with children's emotional and behavioral problems (Xiao, Tang, Deng, \& Pan, 2007). Depression was chosen because it can be predicted by parental and job burnout simultaneously (e.g., Kawamoto et al., 2018).

The Parental Burnout Assessment (PBA; Roskam et al., 2018) is considered the standard of parental burnout because of its background and psychometric properties, and it is a free assessment tool. In the work of Roskam et al. (2018), the PBA contained four factors, i.e., exhaustion due to the parental role, emotional distancing from one's children, the feeling of being fed up, and contrast to previous self. However, in the Chinese version of the PBA, because of the high correlation, the four subfactors could be combined into one (Cheng et al., 2020). As noted by Mikolajczak et al., (2019b), to assess the distinctiveness of parental and job burnout requires investigating burnout in two clearly differentiated but equally important contexts, using validated and structurally similar instruments. Meanwhile, the Chinese Maslach Burnout Inventory (CMBI; Li, Zhang, \& Zhao, 2005) contains three dimensions, i.e., emotional exhaustion, depersonalization, and reduced sense of personal accomplishment. Schaufeli (2017) indicated that, as the core symptom of job burnout, emotional exhaustion is context-free, universal, and not restricted to a particular area or cultural context. Which suggested that the emotional exhaustion was the most representative factor of job burnout. Therefore, the present study using the single-factor PBA, with emotional exhaustion representing job burnout, to examine the distinction between parental and job burnout.

\section{Method}

\section{Participants}

Participants from Study 1 were eighth-grade students (from a middle school located in an urban area in central China) and their parents. Envelopes containing three questionnaires (one for the student and one for each parent) were distributed to 614 students during class. They were told the purpose of the questionnaire was family relationship survey, and they were voluntary to participation and answer. It would not cause any loss if they do not want to participate the survey and can stop answering at any time. Students were asked to complete the questionnaire at home and to bring the completed forms back to school. Father or mother without job were removed from the sample, and the analyses was conducted only with working parents. In total, 597 students, 458 fathers, and 531 mothers completed the questionnaire. The student sample comprised 312 boys and 293 girls, and the average age was 13.9 years $(S D=0.63)$. The average age of the fathers was 41.9 years $(S D=4.18)$ and that of the mothers was 40.9 $(S D=4.09)$. This survey was approved by the Research Ethics Committee of the of the authors' academic institution.

\section{Measures}

\section{Parental Burnout}

Parental burnout was measured using the Chinese version of the Parental Burnout Assessment (PBA; Cheng et al., 2020). This was translated from the English version of Roskam et al. (2018), and had satisfactory reliability and validity. It consisted of 21 items, and each item was rated using a seven-point Likert scale, ranging from 1 ("Completely inconsistent") to 7 ("Completely consistent"), with a higher score representing higher burnout. An example was "I feel as though I've lost my direction as a dad/mum"; the 
Cronbach's $\alpha$ was $0.916(\omega=0.916)$ for fathers' responses and $0.927(\omega=0.927)$ for mothers' responses.

\section{Emotional Exhaustion}

Emotional exhaustion was assessed by five items of the emotional exhaustion factor from the CMBI, which had previously been determined to have adequate reliability and validity for assessing job burnout in Chinese samples (Li et al., 2005). This measurement consisted of five items, each rated using a seven-point Likert scale, ranging from 1 ("Completely inconsistent") to 7 ("Completely consistent"), with a higher score representing higher burnout. An example was "I feel burned out from my work"; the Cronbach's $\alpha$ was $0.772(\omega=0.775)$ for fathers' responses and $0.737(\omega=0.752)$ for mothers' responses.

\section{Depression}

Depression was measured using the Chinese version of the Self-rating Depression Scale (Liu et al. 1994). This consisted of 20 items, each rated using a four-point Likert scale, ranging from 1 ("A little of the time") to 4 ("Most of the time"), with a higher score representing higher depression. An example was "I feel down-hearted and blue"; the Cronbach's $\alpha$ was 0.759 $(\omega=0.754)$ for fathers' responses and $0.759(\omega=0.762)$ for mothers' responses.

\section{Job Satisfaction}

Job satisfaction was measured using the short version of a satisfaction instrument (Schreisheim \&Tsui, 1980). This measurement consisted of six items, each rated using a five-point Likert scale, ranging from 1 ("Strongly disagree") to 5 ("Strongly agree"), with a higher score representing higher satisfaction. An example was "How satisfied are you with the nature of the work you perform?"; the Cronbach's $\alpha$ was $0.901(\omega=0.901)$ for fathers' responses and $0.884(\omega=$ 0.884 ) for mothers' responses.

\section{Children's Anxiety}

Children's anxiety was measured using the Chinese version of the Self-Rating Anxiety Scale (Wu, 1990). This measurement consisted of 20 items, each rated using a four-point Likert scale, ranging from 1 ("A little of the time") to 4 ("Most of the time"), with a higher score representing a higher level of anxiety. An example was "I feel more nervous and anxious than usual"; the Cronbach's $\alpha$ was $0.770(\omega=0.766)$.

\section{Other Measures}

Demographic items, including students' gender and age, as well as parents' age and occupation, were recorded.

\section{Data Analysis}

Data analyses were conducted by R 3.6.1 for windows and AMOS 23.0. First, the Exploratory factor analysis (EFA) and confirmatory factor analysis (CFA) were conducted to confirm whether the parental burnout and the job burnout were loading on different factors. Then, correlation analyses were conducted to preliminarily examine the correlations between the burnout and the consequence variables. Structural equation model (SEM) was also conducted to further examined the relationship between consequences with parental or job burnout.

\section{Results}

\section{Preprocessing of the Data}

Before conducting the EFA, all the answers with missing data were removed from the dataset because most of the questionnaires were unanswered or the questionnaire was blank. And the Welch's test was conducted between the removed and kept data groups. There were no significant differences between the children's age $(t=-0.44, d f=266.19, p=0.66)$, gender $(t=0.28, d f=402.86, p=0.78$ ), and the parents' age (for fathers: $t=0.60, d f=1.00, p=0.66$; for mothers: $t=-0.08, d f=$ $6.01, p=0.94)$. These results suggested that removing the incomplete questionnaires did not bias our results.

The descriptive statistics and correlation matrix are shown in Table 1. Due to the differences in gender roles existing in China, the Welch tests were conducted with all measurements between the samples of fathers and mothers, and the result showed that there were no significant differences between fathers and mothers on job burnout $(t=-0.72, d f=775.55$, $p=.48)$, depression $(t=-1.05, d f=798.12, p=.29)$, and job satisfaction $(t=-1.73, d f=874.55, p=.09)$. However, the level of parental burnout were different between the samples of father and mother $(t=-2.40, d f=843.77, p=.02)$, which suggested mothers sample reported higher level of parental burnout.

Furthermore, a multi-group CFA was conducted to examine the gender invariance of the PBA. Two groups (father and mother) and three models were set. The three models included the unconstrained model, the constrained measurement weight as same, and the constrained structural covariances as same. Results showed that there were no significant differences between the unconstrained model and the constrained measurement weight model $\left[\Delta \chi^{2}(20)=29.94, p=0.08\right]$, which suggested that the factor loading was the same between the two 
Table 1 Descriptive statistics and correlation matrix for Study 1

\begin{tabular}{|c|c|c|c|c|c|c|c|c|c|c|c|c|c|c|c|c|}
\hline & & $M$ & $S D$ & (1) & & (2) & & (3) & & (4) & & (5) & & (6) & (7) & (8) \\
\hline (1) & Fathers' PB & 1.64 & 0.87 & & & & & & & & & & & & & \\
\hline (2) & Fathers' depression & 1.85 & 0.37 & 0.39 & $* *$ & & & & & & & & & & & \\
\hline (3) & Fathers'JB & 3.56 & 0.98 & -0.11 & $*$ & -0.29 & $* *$ & & & & & & & & & \\
\hline (4) & Mothers' PB & 1.79 & 0.96 & 0.49 & $* *$ & 0.16 & $* *$ & -0.02 & & & & & & & & \\
\hline (5) & Mothers' depression & 1.88 & 0.37 & 0.29 & $* *$ & 0.43 & $* *$ & -0.11 & $*$ & 0.43 & $* *$ & & & & & \\
\hline (6) & Mothers' JS & 3.67 & 0.91 & -0.05 & & -0.15 & $* *$ & 0.34 & $* *$ & -0.20 & $* *$ & -0.28 & $* *$ & & & \\
\hline (7) & Children's gender & 1.48 & 0.50 & -0.05 & & -0.01 & & 0.05 & & -0.08 & & -0.03 & & 0.01 & & \\
\hline (8) & Children's age & 13.83 & 0.80 & -0.01 & & 0.05 & & 0.09 & & 0.02 & & 0.02 & & -0.03 & -0.07 & \\
\hline (9) & Children's anxiety & 1.86 & 0.45 & 0.22 & $* *$ & 0.18 & $* *$ & -0.01 & & 0.14 & $* *$ & 0.16 & $* *$ & -0.04 & 0.08 & 0.08 \\
\hline
\end{tabular}

***Note: $p<0.01 ; p<0.05$. SD, standard deviation

groups. There were no significant differences between the unconstrained model and the constrained structural covariances model $\left[\Delta \chi^{2}(21)=30.14, p=0.09\right]$, which suggested that the factor covariances were the same between the two groups. In general, the factor structures were invariant between the two groups.

\section{Factorial Distinctiveness of Parental and Job Burnout}

EFA was conducted on the fathers' answers. Parallel analysis and the minimum average partial (MAP) method were conducted on the 26 burnout items (job burnout, five items; parental burnout, 21 items). Both parallel analysis and MAP suggested a two-factor structure. Using maximum likelihood estimation and oblimin rotation, the first factor contained 21 items of parental burnout and the second factor contained five items of emotional exhaustion.

EFA was also conducted on mothers' answers. When parallel analysis and the MAP method were conducted on the 26 burnout items, however, parallel analysis suggested a threefactor structure and MAP suggested a two-factor structure. Using maximum likelihood and oblimin rotation, when a three-factor structure was set, the item "I have the impression that I'm not myself anymore when I'm interacting with my child(ren)" was dropped because the factor loading was under 0.40. Parental burnout was separated into two factors and the correlation between the two factors was 0.716 . The other five items of emotional exhaustion were loaded onto the third factor. When a two-factor structure was set, the first factor contained 21 items of parental burnout, and the second factor contained five items of emotional exhaustion. These results generally supported that parental burnout and job burnout were different concept and loaded on separated factors. The results were shown in Table 2.

Furthermore, to further support the result of EFA, the CFA was conducted on half of the sample which were selected randomly. When set the parental burnout and job burnout items loading on the different factor, the model showed acceptable goodness fit of the data (Fathers: $\chi^{2}=1056.886$, $d f=283, p<0.01$, the Comparative Fit Index $(\mathrm{CFI})=.903$, the

Table 2 Factor loadings of parental and job burnout for fathers' and mothers' answers

\begin{tabular}{|c|c|c|c|c|c|c|}
\hline \multirow[b]{2}{*}{ Items } & \multicolumn{3}{|c|}{ Fathers' answer } & \multicolumn{3}{|c|}{ Mothers' answer } \\
\hline & Factor1 & Factor2 & $\mathrm{h} 2$ & Factor1 & Factor2 & h2 \\
\hline PBA1 & 0.408 & 0.236 & 0.292 & 0.444 & 0.144 & 0.272 \\
\hline PBA2 & 0.520 & 0.185 & 0.374 & 0.531 & 0.203 & 0.415 \\
\hline PBA3 & 0.645 & -0.028 & 0.404 & 0.699 & 0.062 & 0.529 \\
\hline PBA4 & 0.656 & 0.047 & 0.454 & 0.689 & 0.048 & 0.504 \\
\hline PBA5 & 0.775 & -0.073 & 0.565 & 0.756 & -0.060 & 0.537 \\
\hline PBA6 & 0.792 & -0.147 & 0.566 & 0.798 & -0.162 & 0.554 \\
\hline PBA7 & 0.602 & 0.006 & 0.365 & 0.465 & 0.199 & 0.334 \\
\hline PBA8 & 0.606 & 0.096 & 0.418 & 0.656 & 0.152 & 0.538 \\
\hline PBA9 & 0.621 & 0.003 & 0.388 & 0.603 & 0.226 & 0.529 \\
\hline PBA10 & 0.694 & -0.006 & 0.479 & 0.632 & 0.010 & 0.404 \\
\hline PBA11 & 0.793 & 0.024 & 0.642 & 0.806 & 0.027 & 0.668 \\
\hline PBA12 & 0.703 & 0.109 & 0.562 & 0.802 & -0.005 & 0.641 \\
\hline PBA13 & 0.604 & -0.092 & 0.333 & 0.492 & -0.034 & 0.229 \\
\hline PBA14 & 0.689 & -0.022 & 0.465 & 0.737 & -0.042 & 0.519 \\
\hline PBA15 & 0.605 & 0.163 & 0.463 & 0.639 & 0.065 & 0.448 \\
\hline PBA16 & 0.707 & -0.021 & 0.489 & 0.766 & -0.147 & 0.513 \\
\hline PBA17 & 0.713 & -0.118 & 0.461 & 0.648 & -0.010 & 0.415 \\
\hline PBA18 & 0.688 & 0.071 & 0.514 & 0.731 & 0.047 & 0.566 \\
\hline PBA19 & 0.663 & 0.038 & 0.459 & 0.652 & 0.051 & 0.456 \\
\hline PBA20 & 0.662 & -0.005 & 0.435 & 0.716 & -0.082 & 0.470 \\
\hline PBA21 & 0.606 & -0.009 & 0.364 & 0.678 & -0.067 & 0.426 \\
\hline EE1 & 0.015 & 0.594 & 0.359 & 0.067 & 0.563 & 0.353 \\
\hline EE2 & 0.073 & 0.503 & 0.284 & 0.073 & 0.468 & 0.253 \\
\hline EE3 & 0.018 & 0.764 & 0.595 & -0.087 & 0.855 & 0.675 \\
\hline EE4 & -0.089 & 0.456 & 0.187 & -0.056 & 0.699 & 0.458 \\
\hline EE5 & 0.160 & 0.529 & 0.366 & 0.104 & 0.471 & 0.274 \\
\hline
\end{tabular}

Note: The numbers in blank are factor loadings. $P B A$ Parental Burnout Assessment, EE emotional exhaustion. 
Root Mean Square Error of Approximation $($ RMSEA) $=.067$, the Standardized Root Mean Square Residual $($ SRMR) $=.047$; Mothers: $\chi^{2}=828.75, d f=283, p<0.01, \mathrm{CFI}=.925$, RMSEA $=.056$, SRMR $=.045$ ). These results further supported that items of parental burnout and job burnout belong to different structure.

\section{Differential Outcomes of Parental and Job Burnout}

To examine the distinctive outcome variables of job and parental burnout, a SEM was performed. Fathers' parental burnout and emotional exhaustion were independent variables, and job satisfaction, fathers' depression, and children's anxiety were dependent variables. When we set all of the paths in the model, some paths were not significant. Then, the nonsignificant paths were removed from the model and the results were as shown in Fig. 1 (using the maximum likelihood method).

The model showed an acceptable fit of the data $\left(\chi^{2}=21.26\right.$, $d f=5, p<0.05$; the CFI $=0.917$, the RMSEA $=0.073$. As can be seen in Fig. 1, fathers' parental burnout showed a positive relationship with their children's anxiety $(\beta=0.23, p<0.01)$ and their own depression $(\beta=0.31, p<0.01)$. Fathers' emotional exhaustion had a positive correlation with their own depression $(\beta=0.27, p<0.01)$, and a negative relation with their job satisfaction $(\beta=-0.27, p<0.01)$, which generally met our expectations.

The same model was conducted on mothers' answers, and also showed an acceptable fit for the data $\left(\chi^{2}=24.62, d f=5\right.$, $p<0.001 ; \mathrm{CFI}=0.919$, RMSEA $=0.080)$. Mothers' parental burnout had a positive correlation with their children's anxiety $(\beta=0.13, p<0.01)$ and their own depression $(\beta=0.35$, $p<0.01)$. Meanwhile, mothers' emotional exhaustion had a positive correlation with their own depression $(\beta=0.25$, $p<0.01)$ and a negative correlation with their job satisfaction ( $\beta=-0.28, p<0.01)$, which also met our expectations. The results are shown in Fig. 2.

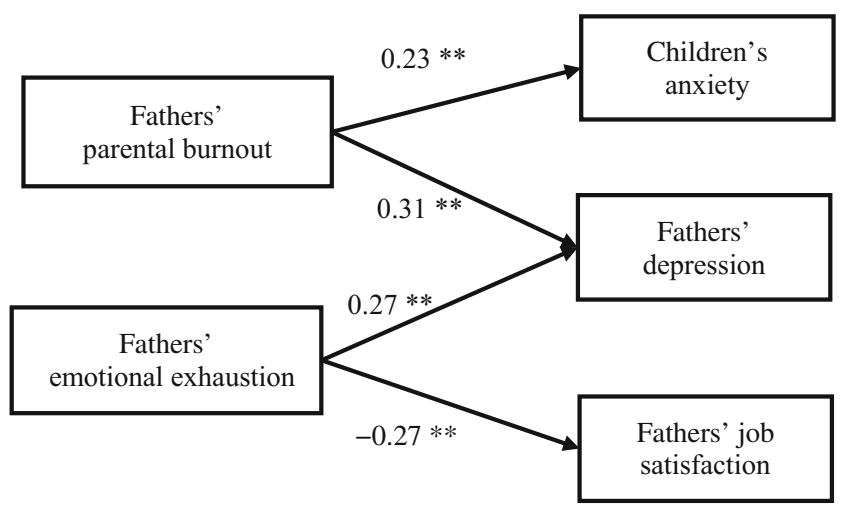

Fig. 1 Results of the SEM of fathers' answers in Study 1. Note: ** $\mathrm{p}<$ 0.01

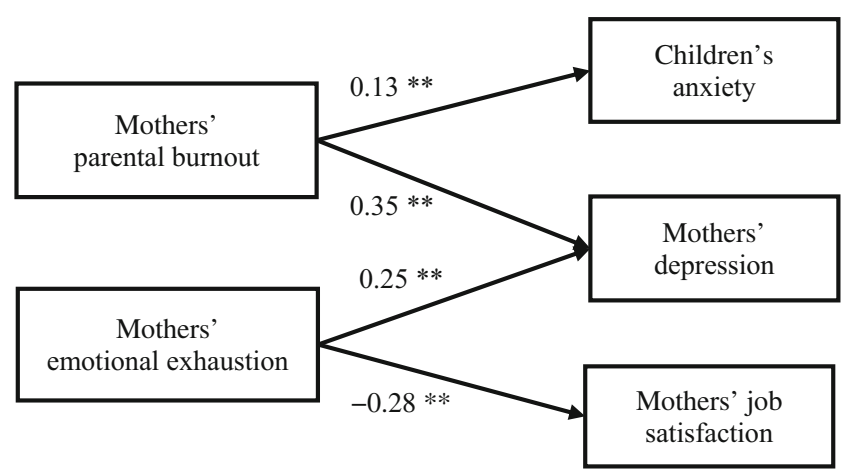

Fig. 2 Results of the SEM of mothers' answers in Study 1. Note: ** $p<$ 0.01

\section{Discussion}

In view of the important and rapid changes in recent decades, the environment of parenting was also different from traditional ways. Parents may experience increased parental stress to bring up their children up healthy, secure, and successfully. While the topic of parental burnout has received considerable attention, whether parental burnout is distinct from related constructs still remains further discussion. In Study 1, using the single-factor measurement of parental and job burnout (represented by emotional exhaustion), the distinction between them was examined. Specifically, the factorial distinctiveness and the different outcomes were examined, and the results generally supported our expectations.

Firstly, in line with previous study (Roskam et al., 2018), the factor structure of PBA was same in both samples of fathers and mothers, however, the levels of parental burnout were different. In which mothers' parental burnout was higher than that of fathers. This result suggested that although the traditional perception of "men work outside; women perform housework at home" were changing, mothers still have to undertake more parenting works than fathers and result in a higher level of parenting burnout. Furthermore, mothers' job burnout did not show statistical different from working fathers. With an increasing number of mothers working fulltime, they may suffer from the parental burnout and job burnout simultaneously. Researchers and practitioners need to pay more attention to working mothers physical and mental health problems.

Secondly, the two-factor structure that separated parental and job burnout into different factors was generally supported in the EFA and CFA, in both fathers and mothers. Which supported that parental burnout and job burnout were two different conception and the burnout was a context-bounded phenomenon (Bakker et al., 2000). Somehow, mothers' answers also suggested a three-factor structure in which parental burnout was divided into two factors. This result may suggest that 21 items loading on one factor-the single-factor structure of PBA- 
was not stable. Further study needs to further revise the questionnaire and improve the quality of measurement. A possible solution was to revise a short version of PBA. Because the long questionnaire may increase the burden of participants and may decrease the response rates, especially in the longitudinal study. Considering that 23 items was still too long, Finnish researchers revised a 5-item version of $\mathrm{PBA}$, and the 5 items were longing to a single factor (Aunola et al., 2020). Therefore, further study may considering revise a Chinese short version of PBA.

Thirdly, parental and job burnout were able to simultaneously predict depression, which is in line with the results of a prior study (e.g., Kawamoto et al., 2018). In addition, parental burnout could have a more pronounced effect on the parenting-related outcome variable of children's anxiety, whereas emotional exhaustion could have a more pronounced effect on the job-related outcome variable of job satisfaction. These results not only support the work of Mikolajczakl et al. (2019b), but also added further evidence of the distinction between parental and job burnout in China.

\section{Study 2: Infer the Direction Between Parental and Job Burnout}

In Study 1, the structural and conceptual differences between parental and job burnout were examined. However, our results do not mean they co-occurred. According to the theory of spillover and compensation (Staines, 1980) and the theory of conservation of resources (Hobfoll, 1989) in work and the family domain, when someone does not have enough resources to cope with family (work) demands, it may result in a lack of resources to handle the work (family) demand. Therefore, further discussion is still needed to determine how they influence each other. To that end, a three-wave longitudinal design and a cross-lag model were established to infer the direction of the relationship.

\section{Participants}

We tracked the parents from Study 1. Surveys on parental burnout and emotional exhaustion were conducted one and 2 months later. All questionnaires with missing data were removed from the sample, because either most of the questions were unanswered or the questionnaire was blank. Furthermore, because some participants only completed the survey once or twice, their data were also deleted. In total, 109 fathers and 144 mothers completed all three questionnaires. The average age of the fathers was 41.6 years $(S D=4.00)$ and that of the mothers 40.4 years $(S D=3.98)$.

\section{Measures}

The measures of parental burnout and emotional exhaustion were the same as in Study 1. As for parental burnout, the Cronbach's $\alpha$ was $0.916,0.956$, and 0.976 at Times 1,2 , and 3 for the fathers' responses, and 0.927, 0.952, and 0.976 at Times 1, 2, and 3 for the mothers' responses. As regards emotional exhaustion, the Cronbach's $\alpha$ was 0.772 , 0.773 , and 0.804 at Times 1,2 , and 3 for the fathers' responses, and $0.737,0.755$, and 0.813 at Times 1,2 , and 3 for the mothers' responses.

\section{Data Analysis}

Data analyses were conducted by SPSS 23.0 and AMOS 23.0. The descriptive statistics and correlation analyses were conducted to preliminarily examine the correlations between parental and job burnout. Then a cross-lag model was conducted to further examined our expectations.

\section{Results}

Because of the low response rate, before further data analysis, Welch's test was conducted between the dropped and retained data groups. There were no significant differences between them in terms of age (for fathers: $t=0.95, d f=210.74, p=$ 0.35 ; for mothers: $t=1.77, d f=319.74, p=0.08$ ). These results suggest that removing the incomplete questionnaires did not significantly bias our results.

The descriptive statistics and correlation matrix are shown in Tables 3 and 4. In order to infer the direction of the relationship between parental and job burnout, a cross-lag model was established, and the results of fathers' answer were shown in Fig. 3. The model showed an acceptable fit of data $\left(\chi^{2}=\right.$ $19.98, d f=4, p<0.01 ; \mathrm{CFI}=0.943$, RMSEA $=0.084)$. As can be observed in Fig. 3, fathers' parental burnout at Time 1 showed a significantly positive relation with emotional exhaustion at Time $2(\beta=0.19, p<0.01)$, and fathers' parental burnout at Time 2 consistently showed a significantly positive relation with emotional exhaustion at Time $3(\beta=0.16$, $p<0.05)$. Furthermore, fathers' emotional exhaustion at Time 1 showed a significantly positive relation with parental burnout at Time $2(\beta=0.27, p<0.01)$. However, the relation between fathers' emotional exhaustion at Time 2 and parental burnout at Time 3 was not significant $(\beta=0.08$, n.s.). In general, fathers' parental burnout had a consistent effect on their job burnout.

The same model was conducted on mothers' answers, and the results were shown in Fig. 4. The model showed an acceptable fit of data $\left(\chi^{2}=26.20, d f=4, p<0.01 ; \mathrm{CFI}=0.938\right.$, RMSEA = 0.099). As an be observed in Fig. 4, mothers' parental burnout at Time 1 showed a significantly positive relation with emotional exhaustion at Time $2(\beta=0.16, p<0.01)$, 
Table 3 Descriptive statistics and correlation matrix of fathers' answers in Study 2

\begin{tabular}{|c|c|c|c|c|c|c|c|c|c|c|c|c|c|c|c|c|}
\hline & & $M$ & $S D$ & (1) & & (2) & & (3) & & (4) & & (5) & & (6) & & (7) \\
\hline (1) & Age & 41.92 & 4.39 & 1.00 & & & & & & & & & & & & \\
\hline (2) & T1 PBA & 1.75 & 0.82 & -0.01 & & 1.00 & & & & & & & & & & \\
\hline (3) & T1 EE & 2.95 & 1.38 & 0.00 & & 0.40 & $* *$ & 1.00 & & & & & & & & \\
\hline (4) & T2 PBA & 2.01 & 0.97 & -0.15 & $*$ & 0.27 & $* *$ & 0.30 & $* *$ & 1.00 & & & & & & \\
\hline (5) & T2 EE & 3.00 & 1.31 & -0.06 & & 0.32 & $* *$ & 0.36 & $* *$ & 0.46 & $* *$ & 1.00 & & & & \\
\hline (6) & Т3РВА & 1.88 & 1.23 & -0.03 & & 0.32 & $* *$ & 0.15 & $*$ & 0.36 & $* *$ & 0.24 & $* *$ & 1.00 & & \\
\hline (7) & T3 EE & 3.00 & 1.44 & -0.02 & & 0.29 & $* *$ & 0.36 & $* *$ & 0.30 & $* *$ & 0.40 & $* *$ & 0.42 & $* *$ & 1.00 \\
\hline
\end{tabular}

***Note: $p<0.01 ; p<0.05$. PBA Parental Burnout Assessment, EE emotional exhaustion, T1-T3 Time 1-3

and mothers' parental burnout at Time 2 consistently showed a significantly positive correlation with emotional exhaustion at Time $3(\beta=0.24, p<0.01)$. Moreover, mothers' emotional exhaustion at Time 1 showed a significantly positive relation with parental burnout Time $2(\beta=0.15, p<0.05)$. However, the relationship between mothers' emotional exhaustion at Time 2 and parental burnout at Time 3 was not significant $(\beta=0.00, n . s$.$) . In general, mothers' parental burnout had a$ consistent effect on their job burnout.

\section{Discussion}

Results of study 1 reveal the parental and job burnout were two different structures, however, the causal relationship between them remains further discussion. In the existing literature of work-family relationship, most of them emphasize the effects of workplace on family domain, for instance, workfamily conflict (e.g., Netemeyer, Boles, \& McMurrian, 1996) and work-family enrichment (Greenhaus, \& Powell, 2006) Recently, studies also focused on the effects of family domain on workplaces, for instance, they indicated that the family-work conflict was also existed. From the perspective of conservation of resources theory (COR; Hobfoll, 1989) and spillover effect (family-to-work or work-to-family; Kinnunen, Feldt, Geurts, \& Pulkkinen, 2006), when parents expend too much resources on parenting work, they may also have insufficient resources to cope pressure situation at workplace, result in job burnout. On the contrary, if working parents were exhausted at work, they may also lack of resources coping the parenting pressure.

Therefore, Study 2 was trying to answer the question of what is the causal relationship between parenting and job burnout. Using the cross-lag model, the direction of the relationship between parental and job burnout was explored. In general, the effect of previous parental burnout on later job burnout was stronger than the effect of previous job burnout on later parental burnout in both fathers and mothers. This means that the overflow direction is from the family domain to the work domain. In other words, if parents do not have enough resources to cope with problems that occur in the family, it affects their work life. This may because influence of traditional Chinese perception "Cultivate the self, regulate the family, govern the state, then lead the world to peace". Which means those who wish to have great achievement must first cultivate their own characters and then manage their families, therefore, Chinese may more emphasize the effect of family than work. However, whether this effect could replicate in other Confucian culture countries (e.g., Japan and Korea) and western countries, future studies should collect data from these countries and make further discussion.

Table 4 Descriptive statistics and correlation matrix of mothers' answers in Study 2

\begin{tabular}{|c|c|c|c|c|c|c|c|c|c|c|c|c|c|c|c|c|}
\hline & & M & SD & (1) & & (2) & & (3) & & (4) & & (5) & & (6) & & (7) \\
\hline (1) & Age & 40.84 & 4.13 & 1.00 & & & & & & & & & & & & \\
\hline (2) & T1 PBA & 1.94 & 0.91 & 0.13 & $*$ & 1.00 & & & & & & & & & & \\
\hline (3) & T1 EE & 3.02 & 1.38 & -0.06 & & 0.41 & $* *$ & 1.00 & & & & & & & & \\
\hline (4) & T2 PBA & 2.08 & 0.99 & 0.12 & $*$ & 0.41 & $* *$ & 0.29 & $* *$ & 1.00 & & & & & & \\
\hline (5) & T2 EE & 3.07 & 1.29 & -0.01 & & 0.26 & $* *$ & 0.34 & $* *$ & 0.37 & $* *$ & 1.00 & & & & \\
\hline (6) & T3 PBA & 1.93 & 1.24 & 0.03 & & 0.39 & $* *$ & 0.19 & $* *$ & 0.40 & $* *$ & 0.15 & $*$ & 1.00 & & \\
\hline (7) & T3 EE & 3.05 & 1.46 & -0.01 & & 0.26 & $* *$ & 0.32 & $* *$ & 0.34 & $* *$ & 0.35 & $* *$ & 0.49 & $* *$ & 1.00 \\
\hline
\end{tabular}

***Note: $p<0.01 ; p<0.05$. PBA Parental Burnout Assessment, EE emotional exhaustion, T1-T3 Time 1-3 
Fig. 3 Results of fathers' parental burnout and emotional exhaustion in study 2. Note: $* * p<0.01$; $* p<0.05$

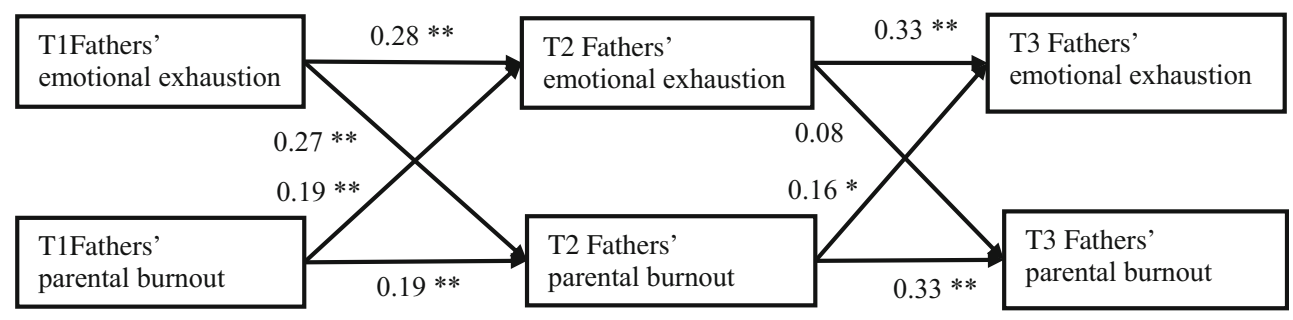

\section{General Discussion}

The present study aimed to determine the relationship between parental and job burnout. Specifically, the factorial distinctiveness of parental and job burnout and their outcomes were examined. Furthermore, the direction of the relationship between parental and job burnout was explored using a cross-lag model.

In general, the results supported our predictions. In Study 1, parental and job burnout were loaded on two different factors in both fathers and mothers. Both of parental and job burnout could predict depression; moreover, parental burnout could significantly predict children's anxiety, while job burnout could significantly predict job satisfaction. These results additionally support the fact that parental and job burnout are different concepts. In Study 2, there was a stronger prediction effect from parental burnout to job burnout than in the reverse direction. These results were repeatedly supported by both fathers' and mothers' answers.

Research on parental burnout has received considerable attention and has achieved great advances. However, these studies are mostly concentered on Western countries (e.g., Roskam et al., 2017; Sorkkila \& Aunola, 2020). Some other regions have taken note of the phenomenon and have begun to research it (e.g., Kawamoto et al., 2018). In general, though, the research performed in Eastern cultures is insufficient. The present study provided research on a Chinese sample, and enriched the development of the parenting burnout theme in an Oriental culture. It not only provided new evidence of the difference between parental and job burnout, but also found that there may be a different factor structure of the PBA. Prior studies on parental burnout used the original four-factor structure (Roskam et al., 2018), or the one-factor structure in the Chinese version of the PBA (Cheng et al., 2020). In the present study, we found that one factor structure may not stable. Future research needs to further focus on improving the reliability and validity of the measurement in China, or using an inductive method to develop a Chinese version parental burnout measurement. Furthermore, based on the content of the measurement, future research could also compare the similarities and differences of parental burnout between the the Eastern and Western culture. Thereby, providing a theoretical basis and interventional guidance for the reduction of parenting burnout and for the improvement of parenting quality in different countries.

Prior studies mainly focused on mothers' parental burnout, and thus most of the samples were mothers only. Even if the studies contained both fathers and mothers, most of them combined fathers and mothers into one sample (e.g., LeVigouroux-Nicolas, Scola, Raes, Mikolajczak, \& Roskam, 2017; Sorkkila \& Aunola, 2020), thus rarely examining the differences between mothers' and fathers' burnout (Roskam et al., 2017). The present study, however, collected family-unit data, divided the sample into fathers and mothers, and viewed the sample of fathers as equally important as the sample of mothers. The results supported the fact that the level of parental burnout was different in fathers and mothers (Roskam et al., 2017); the factor structure may also be different between fathers and mothers. In view of rapid social changes and the change in Chinese perceptions about parenting, a growing number of men have taken on more parenting duties and housework (Zou et al., 2016). Future studies should focus more on the parental role fathers and should provide further evidence to determine whether there are structural differences by gender. This research could provide a basis for identifying and intervening in parental burnout.

Prior studies mainly focused on parental burnout from a parental and family perspective. As two important domains, work and family are closely related and should not be studied separately. In fact, the relationship between work and family has attracted the attention of many scholars, and plenty of research has been done on work-family conflict, work-
Fig. 4 Results of mothers' parental burnout and emotional exhaustion in study 2 . Note: $* *$ $p<0.01$

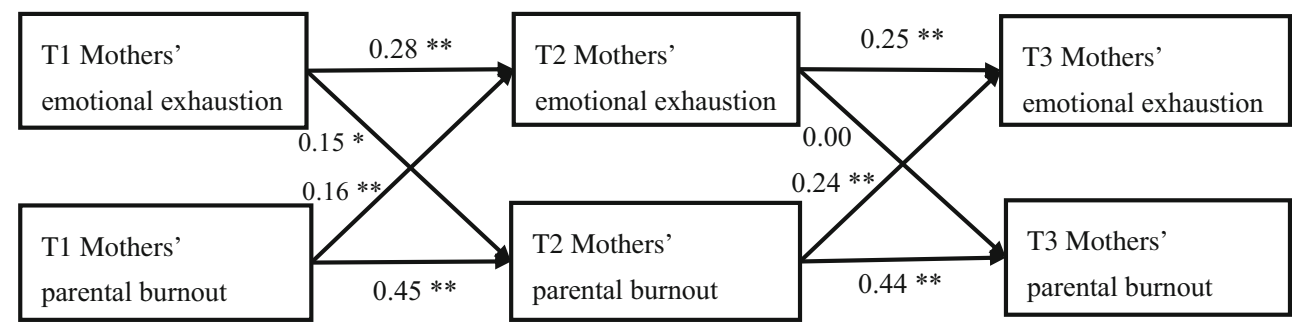


family balance, and work-family enrichment in industrial and organizational psychology (e.g., Michel, Kotrba, Deuling, Clark, \& Baltes, 2011). Based on the spillover and compensation theory (Staines, 1980) and the conservation of resource theory (Hobfoll, 1989) in industrial and organization psychology, the present study paid equal attention to the family and work domains to determine the direction of the relationship between parental and job burnout. In view of the fact that parental burnout is a family-related variable and job burnout is a job- related variable, the present study may also provide a bridge between developmental psychology and industrial and organizational psychology, thereby broadening the range of research on work and family.

\section{Limitations and Future Directions}

Firstly, our sample only included eighth-grade students and their parents from one middle school, which allowed us to collect family-unit longitudinal data. However, the samples were relatively concentrated in age and area, which limits the universality of our conclusions. As noted by Roskam et al. $(2017,2018)$, parental burnout may not be restricted to the parents of school-age children. Thus, future studies should sample from a broader area and should cover a larger age range, from babies to teenagers and their parents.

Secondly, all participants were anonymous. However, the answers relied on a self-reported approach, which may be biased by participants' desire to conform to social norms. Especially, in China, traditional family values emphasize parents' obligations to raise children, to encourage unconditional love for one's children, and to insist that parenting is only right and proper (Shu, 2017). Therefore, some parents may be ashamed to report negative parenting experiences and could selfcensor their answers related to parental burnout. This may be one of the reasons for the high dropout rate in Study 2. Future studies should select more suitable measures and should collect data from different sources-for example, parental burnout could be assessed by spouses and/or children.

Thirdly, the response rate of Study 2 was relatively low. Therefore, the explanation of the results should be more careful. Future research may focus on revising a short version of PBA to reduce the burden of participants and improve the effective response rate.

Author Contributions All authors listed have made a substantial, direct and intellectual contribution to the work. Material preparation, data collection and analysis were performed by Wei Wang, Shengnan Wang, Xia Liu and Yongxin Li. The first draft of the manuscript was written by Wei Wang and all authors commented on previous versions of the manuscript. All authors read and approved the final manuscript.
Funding No funding was received to assist with the preparation of this manuscript.

Data Availability The datasets generated for this study are available on request to the corresponding author.

\section{Declarations}

Ethics Approval The studies involving human participants were reviewed and approved by the Research Ethics Committee of the Institute of Psychology and Behavior, Henan University. Written informed consent to participate in this study was provided by the participants' legal guardian/next of kin.

Conflict of Interest The authors declare that the research was conducted in the absence of any commercial or financial relationships that could be construed as a potential conflict of interest.

Open Access This article is licensed under a Creative Commons Attribution 4.0 International License, which permits use, sharing, adaptation, distribution and reproduction in any medium or format, as long as you give appropriate credit to the original author(s) and the source, provide a link to the Creative Commons licence, and indicate if changes were made. The images or other third party material in this article are included in the article's Creative Commons licence, unless indicated otherwise in a credit line to the material. If material is not included in the article's Creative Commons licence and your intended use is not permitted by statutory regulation or exceeds the permitted use, you will need to obtain permission directly from the copyright holder. To view a copy of this licence, visit http://creativecommons.org/licenses/by/4.0/.

\section{References}

Aunola, K., Sorkkila, M., Tolvanen, A., Tassoul, A., Mikolajczak, M., \&Roskam, I. (2020). Development and validation of the brief parental burnout scale (BPBs). Unpublished manuscript.

Bakker, A. B., Schaufeli, W. B., Demerouti, E., Janssen, P. P. M., Van Der Hulst, R., \& Brouwer, J. (2000). Using equity theory to examine the difference between burnout and depression. Anxiety, Stress \& Coping, 13(3), 247-268.

Cheng, H., Wang, W., Wang, S., Li, Y., Liu, X., \& Li, Y. (2020). Validation of a Chinese version of the parental burnout assessment. Frontiers in Psychology. https://doi.org/10.3389/fpsyg.2020.00321.

Crnic, K., \& Low, C. (2002). Everyday stresses and parenting. In M. H. Bornestein (Ed.), Handbook of parenting volume 5 practical issues in parenting (pp. 243-268). Mahwah, NJ: Lawrence Erlbaum Associates.

Freudenberger, H. J. (1974). Staff burn-out. Journal of Social Issues, 30, $159-165$.

Greenhaus, J. H., \& Powell, G. N. (2006). When work and family are allies: A theory of work-family enrichment. Academy of Management Review, 31(1), 72-92.

Hobfoll, S. (1989). Conservation of resources. American Psychologist, 44, 513-524.

Igawa, J. (2018). An exploratory study on the similarity of "burnout" and “depression” [バンアウトとうつの 似性に関する探索的研究]. Oita University Economic Review, 70, 1-26 (in Japanese).

Kawamoto, T., Furutani, T., \& Alimardan, M. (2018). Preliminary validation of Japanese version of the parental burnout inventory and its relationship with perfectionism. Frontiers in Psychology. https://doi. org/10.3389/fpsyg.2018.00970. 
Kinnunen, U., Feldt, T., Geurts, S., \& Pulkkinen, L. (2006). Types of work-family interface: Well-being correlates of negative and positive spillover between work and family. Scandinavian Journal of Psychology, 47(2), 149-162.

Le Vigouroux, S., Scola, C., Raes, M. E., Mikolajczak, M., \& Roskam, I. (2017). The big five personality traits and parental burnout: Protective and risk factors. Personality and Individual Differences, 119, 216-219.

Li, Y. X., Zhang, K., \& Zhao, G. X. (2005). Confirmatory factor analysis of job burnout. Psychological Exploration, 25, 70-73 (In Chinese).

Liu, X., Dai, Z., Tang, M., Chen, X., Hu, L., \& Wang, A. (1994). Factor analysis of self rating depression scale (SDS). Chinese Journal of Clinical Psychology, 2(3), 151-154 (In Chinese).

Lizano, E. L., \& Barak, M. M. (2015). Job burnout and affective wellbeing: A longitudinal study of burnout and job satisfaction among public child welfare workers. Children and Youth Services Review, 55, 18-28.

Maslach, C., \& Jackson, S. E. (1981). The Maslach burnout inventory. Palo Alto, CA: Consulting Psychologists Press. https://doi.org/10. 1037/t05190-000.

Maslach, C., Schaufeli, W. B., \& Leiter, M. P. (2001). Job burnout. Annual Review of Psychology, 52, 397-422.

Michel, J. S., Kotrba, L., Deuling, J. K., Clark, M. A., \& Baltes, B. (2011). Antecedents of work-family conflict: A meta-analytic review. Journal of Organizational Behavior, 32(5), 689-725. https:// doi.org/10.1002/job.695.

Mikolajczak, M., Brianda, M. E., Avalosse, H., \& Roskam, I. (2018). Consequences of parental burnout: Its specific effect on child neglect and violence. Child Abuse and Neglect, 80, 134-145. https:// doi.org/10.1016/j.chiabu.2018.03.025.

Mikolajczak, M., Gross, J. J., \& Roskam, I. (2019a). Parental burnout: What is it and why does it matter? Clinical Psychological Science., 7, 1319-1329. https://doi.org/10.1177/2167702619858430.

Mikolajczak, M., Gross, J. J., Stinglhamber, F., Norberg, A. L., \& Roskam, I. (2019b). Is parental burnout distinct from job burnout and depressive symptomatology? Clinical Psychological Science, 8, 673-689. https://doi.org/10.1177/2167702620917447.

Nelson, S. K., Kushlev, K., \& Lyubomirsky, S. (2014). The pains and pleasures of parenting: When, why, and how is parenthood associated with more or less well-being? Psychological Bulletin, 140, 846-895.

Netemeyer, R. G., Boles, J. S., \& McMurrian, R. (1996). Development and validation of work-family conflict and family-work conflict scales. Journal of Applied Psychology, 81(4), 400-410.

Pelsma, D. M. (1989). Parent burnout: Validation of the maslach burnout inventory with a sample of mothers. Measurement and Evaluation in Counselling and Development, 22, 81-87. https://doi.org/10. 1080/07481756.1989.12022915.
Pines, A. M. (1987). Sex differences in marriage burnout. Israel Social Science Research, 5, 60-75.

Roskam, I., Raes, M. E., \& Mikolajczak, M. (2017). Exhausted parents: Development and preliminary validation of the parental burnout inventory. Frontiers in Psychology. https://doi.org/10.3389/fpsyg. 2017.001.

Roskam, I., Brianda, M. E., \& Mikolajczak, M. (2018). A step forward in the conceptualization and measurement of parental burnout: The parental burnout assessment (PBA). Frontiers in Psychology. https://doi.org/10.3389/fpsyg.2018.007.

Sánchez-Rodríguez, R., Perier, S., Callahan, S., \& Séjourné, N. (2019). Revue de la littérature relative au burnout parental. Canadian Psychology. https://doi.org/10.1037/cap0000168 (In French).

Schaufeli, W. B. (2017). Burnout: A short socio-cultural history. In S. Neckel, A. K. Schaffner, \& G. Wagner (Eds.), Burnout, fatigue, exhaustion: An interdisciplinary perspective on a modern affliction (pp. 105-127). Cham: Palgrave MacMillan.

Schriesheim, C.; Tsui, A.S. (1980). Development and validation of a short satisfaction instrument for use in survey feedback interventions. Paper Presented at the Western Academy of Management Meeting.

Shu, X. (2017). Word Ploicy. Available online at: http://worldpolicy.org/ 2017/10/16/china-education-and-familybonds/\#: :text=Even\% 20though $\% 20$ bonds $\% 20$ have $\% 20$ weakened,for $\% 20$ financial $\%$ 20and\%20interpersonal\%20support. Accessed 3 Mar 2021.

Sorkkila, M., \& Aunola, K. (2020). Risk factors for parental burnout among Finnish parents: The role of socially prescribed perfectionism. Journal of Child and Family Studies. https://doi.org/10.1007/ s10826-019-01607-1.

Staines, G. L. (1980). Spillover versus compensation: A review of the literature on the relationship between work and nonwork. Human Relations, 33(2), 111-129.

Wu. (1990). Self-rating anxiety scale. Shanghai Archives of Psychiatry, 2(Supplement Issue), 44.

Xiao, C., Tang, Q., Deng, Y., \& Pan, C. (2007). The relation between child psychological abuse/neglect and the parental rearing behaviors. China Journal of Health Psychology, 15(3), 200-202 (In Chinese)

Zhang, J. (2010). An analysis of the stereotype of "men work outside and women perform housework inside" in China. Legal System and Society, 17, 200 (In Chinese).

Zou, K., Wu, X., \& Liu, C. (2016). The effect of maternal gatekeeping: Concept, theory and prospective. Journal of Beijing Normal University (Social Sciences), 6, 41-49 (In Chinese).

Publisher's Note Springer Nature remains neutral with regard to jurisdictional claims in published maps and institutional affiliations. 